\title{
Adhesive Property and Phase Separation of Poly(vinyl ethylene-co- 1,4-butadiene) with Rosin Resin Mixtures
}

\author{
Seiichi KaWahara and Saburo AkiYama \\ Laboratory of Chemistry, Faculty of General Education, \\ Tokyo University of Agriculture and Technology, \\ 3-5-8, Saiwai-cho, Fuchu-shi, \\ Tokyo 183, Japan
}

(Received March 7, 1990)

\begin{abstract}
An early stage of phase separation by spinodal decomposition in mixtures of poly(vinyl ethylene-co-1,4-butadiene) with $70.4 \mathrm{wt} \%$ vinyl ethylene content (V-BR (70.4)) and a rosin resin (Gumrosin X) was investigated by light scattering technique. The V-BR (70.4)/Gumrosin $\mathrm{X}$ mixture was found to exhibit lower critical solution temperature (LCST) behavior. The time evolution of scattered intensity was followed as a function of temperature jump, so that the linearized Cahn-Hilliard's theory was reasonably applied to the spinodal decomposition. As a result of detecting the spinodal decomposition, we predicted that a pressure sensitive adhesive property is related to the phase dispersed structure induced by jumping temperature over the LCST.
\end{abstract}

KEY WORDS Miscibility / Pressure Sensitive Adhesive / Phase Separation

Kinetics / V-BR Plasticizer Mixture / Light Scattering / Lower Critical

Solution Temperature $/$

In researching into mixing polymer physics, of particular interesting is a thermodynamic interpretation of phase separation. Some properties and behavior for polymer mixtures have been elucidated in several papers..$^{1-3}$ In practice, a study of polymer miscibility has made us develop various multicomponent polymers. For instance, dispersity and miscibility of loading one polymer into another polymer play an important role in paints and adhesives.

Pressure sensitive adhesives are, in general, composed of rubbers and plasticizers. In mixtures, the performance of pressure sensitive adhesives is related to the viscoelastic response of bulk adhesives, as well as to surface energies of adhesives and adhered. ${ }^{4}$ Although the mechanism of the high performance of adhesives has not been explained, adhesive properties would be dominated by miscibility of mixtures. Hock ${ }^{5}$ and Wetzel $^{6}$ concluded that, in mixtures of natural rubber and resin, when the elastomer and resin formed a two-phase system after properly combining, the separated disperse phase was effectively a viscous fluid which could wet and flow to intimately contact a surface in a tack. On the other hand, Fukuzawa pointed out that the same tack was caused from reducing viscosity due to an enhancement of miscibility in the mixtures ${ }^{7}$. In regard to block copolymers, such as styrene isoprene-styrene block copolymer (SIS) and styrene-butadiene-styrene block copolymer (SBS), the adhesives were acted on, by phase dispersity and affected by whether the tackifing resin was miscible with polystyrenerich or rubber-rich phase. ${ }^{8}$ Among investigations on the adhesive property in relation with phase morphology, there is little thermodynamic approach in adhesives, and the possibility of thermodynamic interpretation on the basis of phase equilibria in binary mixtures was suggested only by Nishi. ${ }^{9}$

We investigated the miscibility of mixtures in adhesives and the relationship between the 
adhesive property and phase morphology in immiscible mixtures. ${ }^{10-13}$ The miscibility and adhesive properties for mixtures of SIS with Piccolyte and with Piccotex were studied in regard to phase equilibria in quasi-binary mixtures. From results of cloud point measurements, SIS/Piccolyte mixtures exhibited lower critical solution temperature (LCST) phase behavior, whereas SIS/Piccotex mixtures exhibited an upper critical solution temperature (UCST) phase behavior. We concluded, consequently, that the adhesive property at room temperature was better near a critical composition due to large concentration fluctuation. ${ }^{10}$ Thus, in conclusion, micro-dispersed structures under the influence of the concentration fluctuation that resulted in phase separation would enhance the adhesive property.

In our last study, pressure sensitive adhesive property and the miscibility were investigated in mixtures of poly(vinylethylene-co-1,4-butadiene)(V-BR) with several vinyl ethylene (vinyl) contents and some tackifier resins. ${ }^{14}$ It was found that the mixtures with rosin resins were excellent adhesives and that the mixtures of V-BR with $70.4 \mathrm{wt} \%$ vinyl content and Gumrosin X exhibited LCST phase behavior. In this study, the adhesive property of V-BR (70.4)/Gumrosin $\mathrm{X}$ mixtures was studied by a ball tack measurement, based on J. Dow method, and by peel strength at $180^{\circ}$. Furthermore, spinodal decomposition in LCST phase behavior was also investigated by a light scattering technique, with respect to concentration fluctuation.

\section{EXPERIMENTAL}

The polymer sample used in this study was V-BR (70.4) supplied by Nippon Zeon Co., Ltd., V-BR (70.4) $\left(\bar{M}_{w}=232,000, \bar{M}_{w} / \bar{M}_{n}=\right.$ 1.05) was prepared by polymerizing 1,3butadiene in hexane or benzene with a $n$-butyllithium catalyst in combination with diglyme (diethylene glycol dimethyl ether) at $40^{\circ} \mathrm{C}$. A tackifier was Gumrosin X supplied by
Hitachi Kasei Polymer Co., Ltd. The others were Staybelite ester 10 and Foral 105 by Rika Hercules, and Super ester A 115 by Arakawa Chemical Industry Co., Ltd. Mixtures of V-BR (70.4) with Gumrosin X were stirred in $10 \mathrm{wt} \%$ toluene solutions at provided ratio. As-cast films were prepared by casting the solution onto a cover glass (for microscopy). The cast films were dried under vacuum at $40^{\circ} \mathrm{C}$ for a week.

Films used in ball tack and peel strength measurements were prepared by casting $20 \mathrm{wt} \%$ toluene solution onto poly(ethylene terephtarate)(PET) film after being isotropic coated with the mixtures by rolling bar. After the solvent was evaporated at room temperature, the films were dried at $80^{\circ} \mathrm{C}$ for 2 hours. The sample thickness was about $30 \mu \mathrm{m}$. The measurements of tack and peel strength obeyed JIS-z0237. The ball tack experiment, based on Dow method, was carried out at definite angle, $\theta=30^{\circ}$. The peel strength in $180^{\circ}$ was measured using Shimazu IS-2000 with stainless plate.

Light scattering profiles were obtained by OPTEC GP-5 light scattering analytical system. The film on the cover glass, set horizontally on the light scattering stage, was kept at constant temperature for annealing. Radiation from a $\mathrm{He}-\mathrm{Ne}$ gas laser of $632.8 \mathrm{~nm}$ wavelength was vertical to the film specimen. The details of the system were reported in a previous paper. ${ }^{14}$

\section{RESULTS AND DISCUSSION}

$\mathrm{V}-\mathrm{BR}$ is a random copolymer comprised of vinyl ethylene and 1,3-butadiene as monomeric units. In V-BR with about $70 \mathrm{wt} \%$ vinyl content, excellent balance of low rolling resistance and high wet grip for tire materials was revealed. ${ }^{15}$ Thus, it is possible for V-BR with $70 \mathrm{wt} \%$ vinyl content to take advantage of adhesive properties.

As shown in Table I, the tack properties and peel strength of V-BR (70.4)/rosin resin mixtures are suitable for adhesives, compared with commercial pressure sensitive adhesives. 
Table I. Adhesive properties of the V-BR $(70,4) /$ tackfier system

\begin{tabular}{lcccc}
\hline \multirow{2}{*}{ Sample } & \multicolumn{2}{c}{ Ball tack } & Peel strength & \multicolumn{2}{c}{ Film thickness } \\
\cline { 2 - 3 } & ball No. & gf/10 mm & & $\mu \mathrm{m}$ \\
\hline Gumrosin X & 12 & 395 & 30 \\
Staybelite ester & 7 & 372 & & 30 \\
Foral 105 & 14 & 383 & & 30 \\
Super ester A115 & - & 395 & 30 \\
\hline
\end{tabular}

For instance, the tack property and peel strength of cellophane adhesive tape with $52 \mu \mathrm{m}$ thickness are 15 in ball number and 350 in $\mathrm{gf} / 10 \mathrm{~mm}$, respectively. Although a V-BR (70.4)/Super ester A 115 mixture exhibits specific adhesive property, a V-BR (70.4)/ Gumrosin X mixture is of great interest because of phase separation with rising temperature. Thus, we can investigate adhesive properties with concentration fluctuation in relation to phase separation.

Among the mixtures, the peel strength of V-BR (70.4)/Gumrosin $\mathrm{X}$ with various mixing ratios in the mixtures is shown in Figure 1 and Table II. The peel strength increased with increasing Gumrosin X content in the mixtures. The peel strength of mixtures with high Gumrosin X content above $60 \mathrm{wt} \%$ was scattered and then ceased to be evident. In our previous studies, ${ }^{10}$ the maximum tack properties of pressure sensitive adhesives appeared to occur at critical compositions in phase behavior. Therefore, the maximum peel strength of the V-BR (70.4)/Gumrosin X may appear in the vicinity of critical composition. In the customary preparation of adhesive films, they are annealed at about $90^{\circ} \mathrm{C}$ for 1 minute. If the concentration fluctuation with annealing occurs in adhesives, the adhesive properties can be influenced by miscibility and a micro-phase disperse structure to attain maximum value. From this point of view, the thermodynamic interpretation with phase behavior is essential to elucidate the adhesive mechanism.

The phase separated structure in LCST

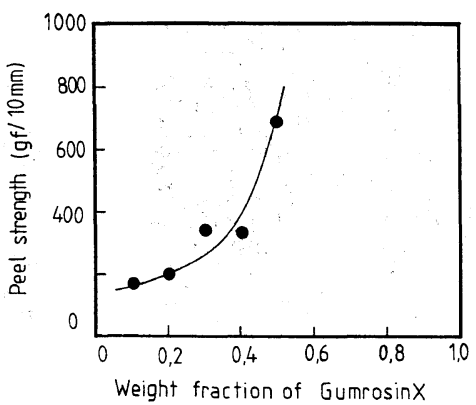

Figure 1. Composition dependence of peel strength for the V-BR (70.4)/Gumrosin X system.

Table II. Composition dependence of pell strength for V-BR (70.4)/Gumrosin X system

\begin{tabular}{cc}
\hline & Peel strength \\
\cline { 2 - 2 } & gf/10 mm \\
\hline $9 / 1$ & 167 \\
$8 / 2$ & 200 \\
$7 / 3$ & 342 \\
$6 / 4$ & 333 \\
$5 / 5$ & 683 \\
\hline
\end{tabular}

phase behavior for the V-BR (70.4)/Gumrosin $\mathrm{X}$ is similar to that of spinodal decomposition, which has the morphological features of unique periodicity and a high level of phase connectivity. A typical highly interconnected two phase morphology with uniform domain size is shown in Figure 2. This phase contrast micrograph was taken after annealing the V-BR (70.4)/Gumrosin X (50/50 by weight) mixture at $100^{\circ} \mathrm{C}$ for 4 hours. Thus the kinetic interpretation of phase separation in V-BR (70.4)/Gumrosin $\mathrm{X}$ would easily allow us to evaluate composition fluctuation. The kinetics of spinodal decomposition in polymer blends and polymer solutions has been studied by many investigators with light scattering apparatus. We also examined the phase separation of the V-BR (70.4)/Gumrosin $\mathrm{X}$ in a similar way. ${ }^{16}$

Some typical time evolutions of scattering curves for V-BR (70.4)/Gumrosin X (50/50) mixtures are depicted in Figure $3 \mathrm{a}-\mathrm{d}$ for 


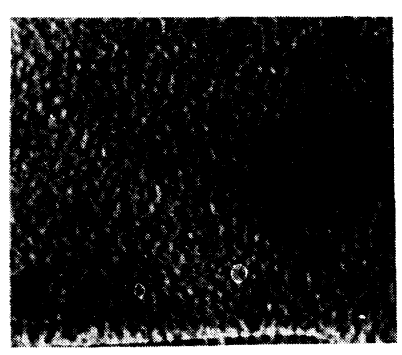

(a)

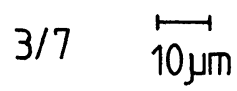

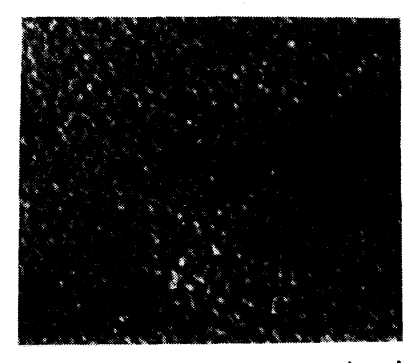

(b) $5 / 5$

$\stackrel{\longmapsto}{10 \mathrm{~mm}}$

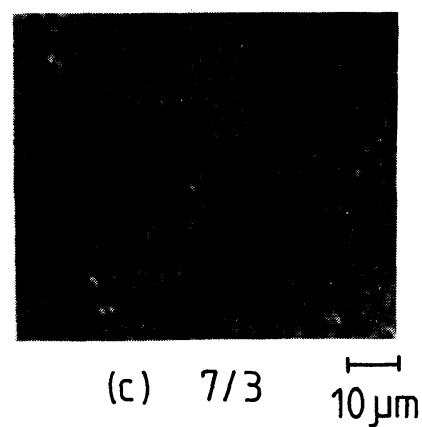

Figure 2. Phase contrast micrographs of V-BR (70.4)/Gumrosin $\mathrm{X}$ at $100^{\circ} \mathrm{C}$ for 4 hours: (a), 3/7; (b), $5 / 5 ;(\mathrm{C}), 7 / 3$ mixtures.

various temperature jumps. When the blend underwent phase separation, a clear scattering maximum developed at a particular angle. As the phase separation proceeded, the scattered intensity at a maximum scattering vector $q_{\mathrm{m}}$ increased immensely with phase separation time. This kind of linear behavior was first reported by Hashimoto et al. ${ }^{17}$ in the spinodal decomposition of poly(styrene) (PS)/poly(vinyl methyl ether) (PVME) blends. A linear regime might persist in the stage of spinodal decomposition. Examination should be made with the so-called linearized Cahn-Hilliard theory ${ }^{18-22}$ in which scattered intensity is predicted to increase exponentially. Its scattered intensity is given by

$$
I(q, t)=I(q, 0) \exp \{2 R(q) t\}
$$

where $R(q)$ is the growth rate of concentration fluctuation having $q, t$ is the time after the initiation of spinodal decomposition, and $\mathrm{q}$ is the magnitude of scattering vector, i.e.,

$$
q=\frac{4 \pi}{\lambda_{0}} \sin \frac{\theta}{2}
$$

where $\lambda_{0}$ is the wave length of light and $\theta$ is the scattering angle in the medium. Although the inadequacy of the Cahn-Hilliard theory was first pointed out by Cook who modified the linear theory by taking into account the effect of thermal fluctuation of a stable solution, we adopt Cahn-Hilliard theory for the initial stage of spinodal decomposition because of existing constant $\left(q_{\mathrm{m}}\right)$ during phase separation. $R(q)$ is given as

$$
R(q)=-M q^{2}\left[\left(\frac{\partial^{2} f}{\partial \phi^{2}}\right)_{0}+\kappa q^{2}\right]
$$

where $M$ is mobility, $\left(\partial^{2} f / \partial \phi^{2}\right)_{0}$ is the second derivative of free energy $(f)$ at the initial composition $\left(\phi_{0}\right)$, and $\kappa$ is a coefficient of composition gradient. $R(q)$ is a quantity that may be determined experimentally by using light scattering.

The time evolution of scattering curves was further analyzed according to eq (1) by plotting logarithmic intensity versus phase separation time for all temperature jumps. Figure 4 shows a typical change in scattered light intensity at various $q$ for the V-BR (70.4)/Gumrosin $\mathrm{X}$ $(50 / 50)$ mixture annealed to an isothermal setting at $100^{\circ} \mathrm{C}$. The intensity increases exponentially with time length for some initial periods. Linear results at initial stage were also obtained for mixtures with other compositions at various temperatures. When the sample was further annealed, the intensity deviated significantly from exponential growth. Although this may be associated with additional processes occurring at late stages of spinodal decomposition, let us consider the concentration fluctuation at the initial stage of spinodal decomposition. From the slopes of the linear expressions in the semilogarithmic plot of 

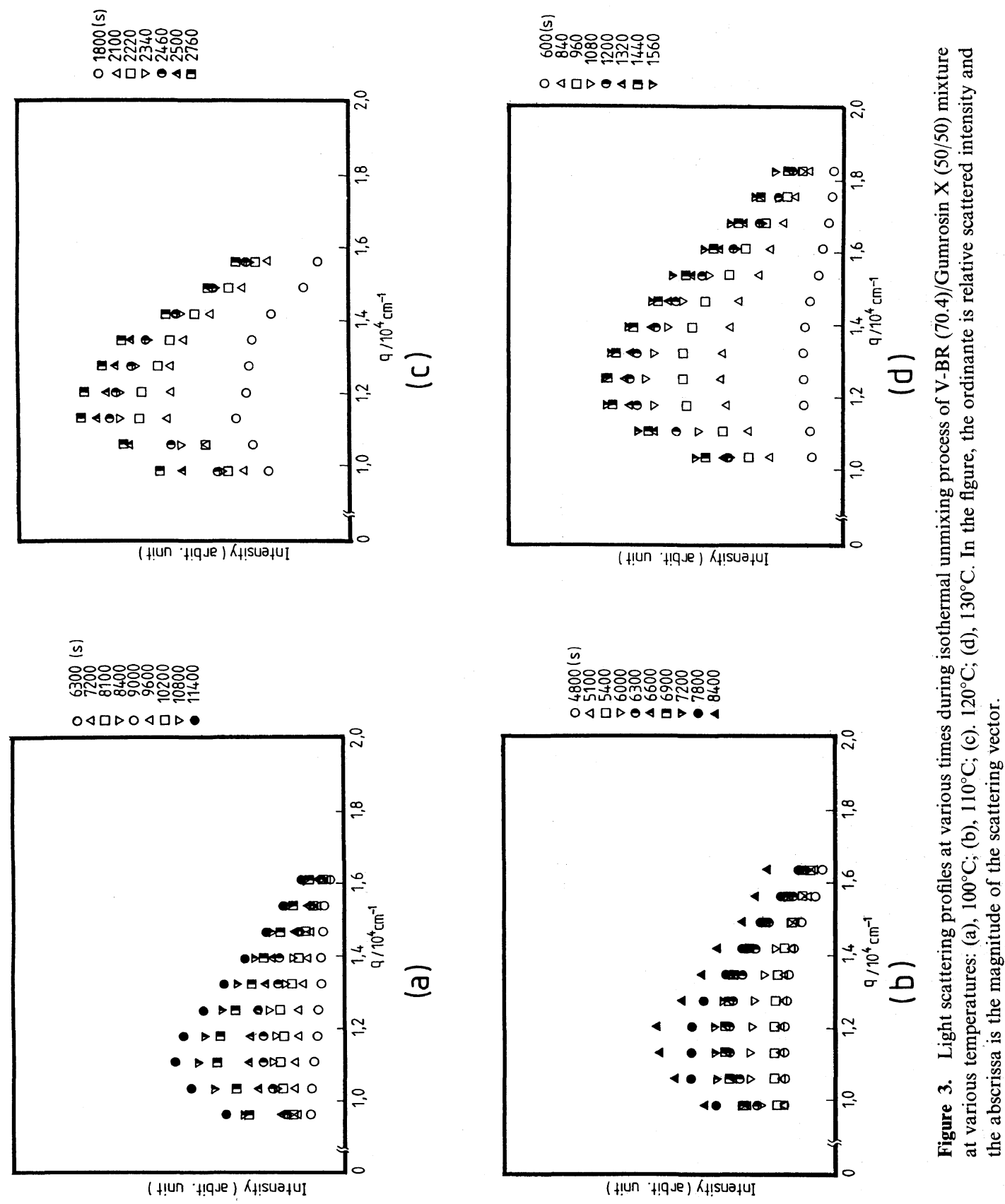

( t!Un 't!qJe) Kł!ISUafuI 


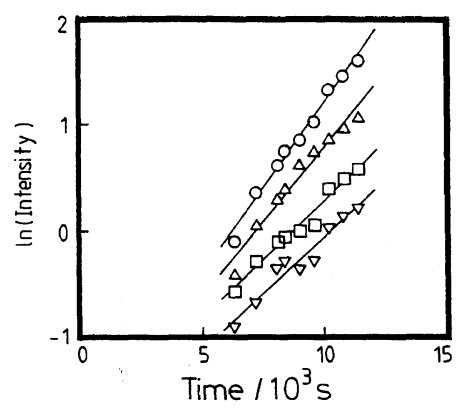

Figure 4. Change in scattered light intensity at various $q$ 's for V-BR (70.4)/Gumrosin X (50/50) mixture annaealed to an isothermal setting at $100^{\circ} \mathrm{C}$, demonstrating the exponential character of $I(t)$ at the initial stage of phase separation: $(\bigcirc), q=1.107 \times 10^{-4} \mathrm{~cm}^{-1} ;(\triangle), 1.395 \times 10^{-4}$ $\mathrm{cm}^{-1} ;(\square), 1.539 \times 10^{-4} \mathrm{~cm}^{-1} ;(\nabla), 1.611 \times 10^{-4} \mathrm{~cm}^{-1}$.

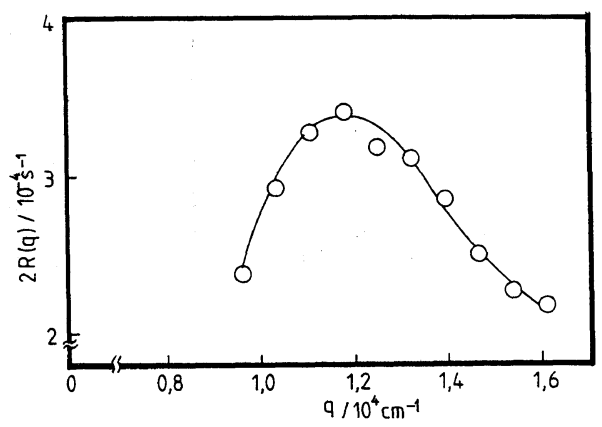

Figure 5. Wavenumer $q$ dependence of the the growth rate $R(q)$ in V-BR (70.4)/Gumrosin X (50/50) mixture at $100^{\circ} \mathrm{C}$. The peak indicates existence of the maximum growth rate $R\left(q_{\mathrm{m}}\right)$.

scattering intensity versus time, $R(q)$ for various $q$ were determined. Figure 5 shows $R(q)$ as a function of $q$. In the context of Cahn's linearized theory, $R(q)$ was shown to have maximum at $q_{\mathrm{m}}$. As shown in Figure 5, there is a single peak in $R(q)$ versus $q$ plot, and the maximum growth rate $R\left(q_{\mathrm{m}}\right)$ is found. Then in accordance with equation (3), $R(q) / q^{2}$ were plotted against $q^{2}$ for the V-BR (70.4)/gumrosin X 50/50 mixture in Figure 6. As expected from eq (3), the plots in the figure yielded straight lines. By the intercept of $R(q) /$ $q^{2}$ at $q^{2}=0$, one can obtain the apparent diffusion coefficient $D_{\text {app }}$, i.e.,

$$
D_{\text {app }}=M\left(\frac{\partial^{2} f}{\partial \phi^{2}}\right)_{0}
$$

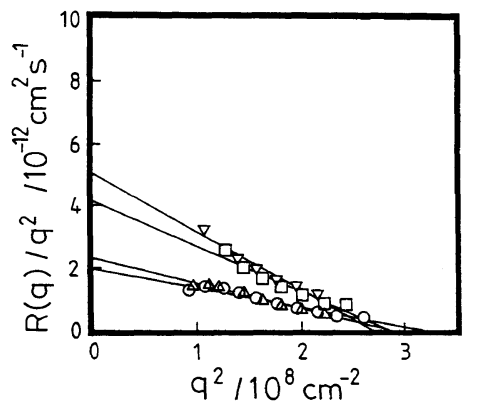

Figure 6. Plot of $R(q) / q^{2} v s . q^{2}$ in V-BR (70.4)/Gumrosin $\mathrm{X}(50 / 50)$ mixture: $(O), 100^{\circ} \mathrm{C} ;(\triangle), 110^{\circ} \mathrm{C} ;(\square), 120^{\circ} \mathrm{C}$; $(\nabla), 130^{\circ} \mathrm{C}$

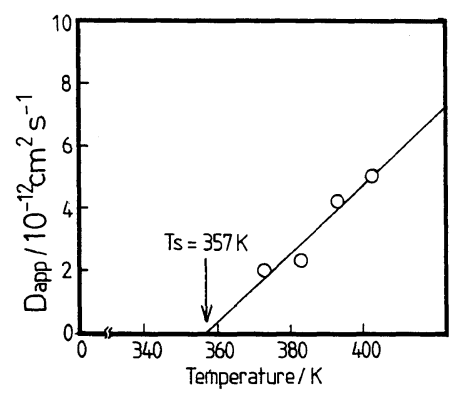

Figure 7. Temperature dependence of apparent diffusion coefficient $D_{\text {app }}$ in V-BR (70.4)/Gumrosin X (50/50) mixture.

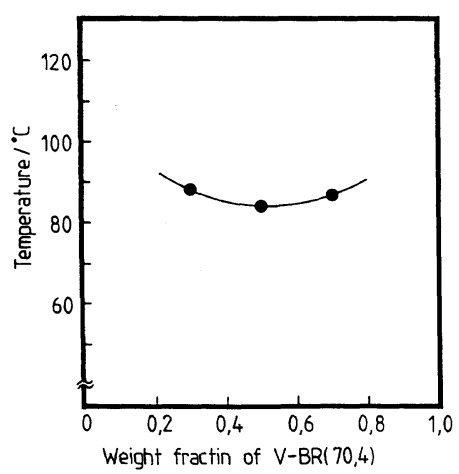

Figure 8. LCST type phase diagram of V-BR (70.4)/ Gumrosin X mixture measured by light scattering method. The closed circles represent calculated spinodal points, and the solid line represents estimated spinodal curves.

From the temperature dependence of $D_{\mathrm{app}}$, a linear relationship between $D_{\text {app }}$ and temperature is shown in Figure 7. The second derivative 
of free energy vanishes at spinodal temperature $T_{\mathrm{s}}$ shown by the arrow in Figure 7 , therefore $T_{\mathrm{s}}$ can be estimated by the intercept of temperature at $D_{\text {app }}=0$. Figure 8 shows $T_{\mathrm{s}}$ as a function of composition, which delineates a concave curve. This type of phase diagram shows LCST phase behavior.

In an industrial process for preparing pressure sensitive adhesives, a net thermal treatment for casting the solvent is carried out at about $90^{\circ} \mathrm{C}$ for 1 minute. In order to take account of the phase dispersity with respect to concentration fluctuation, we may have to inquire into a solvent evaporation proccss in polymer-resin-solvent ternary system. Growth dynamics relative to the concentration fluctuation, which appears to occur with casting the solvent, leads to clarify a phase separated mechanism of mixture. ${ }^{23,24}$ In our study, the growth dynamics of V-BR (70.4)/Gumrosin X mixtures were investigated during isothermal treatment after casting the solvent. Then the spinodal decomposition for the binary mixtures was found at the temperature $80^{\circ} \mathrm{C}$. As the mixtures have been already annealed at $80^{\circ} \mathrm{C}$ for 2 hours, the concentration fluctuation in the binary mixtures may influence the phase dispersity to enhance the adhesive property. We found the spinodal decomposition of the mixtures and then estimated the $T_{\mathrm{s}}$. Thus it is possible to estimate that the adhesive property may be influenced by the dispersed spinodal structure.

\section{CONCLUSION}

The kinetics of phase separation of the V-BR (70.4)/Gumrosin X mixtures were investigated by light scattering techniques. When the mixtures were annealed at isothermal setting temperatures above LCST, the scattered light intensity increased exponentially for some initial periods. By applying the linearized Cahn-Hilliard's theory to the phase separation, spinodal temperatures $T_{\mathrm{s}}$ were estimated at several mixing ratios; $86.4^{\circ} \mathrm{C}(7 / 3), 84.0^{\circ} \mathrm{C}$
$(5 / 5)$, and $88.2^{\circ} \mathrm{C}(3 / 7)$. Then concentration fluctuation would be easily revealed at the temperature above $T_{\mathrm{s}}$. As for investigating the pressure sensitive adhesive property with respect to the phase dispersed structure, one may obtain more precise insight into the adhesive mechanism.

Acknowledgments. The authors express their sincere thanks to Dr. A. Ueda of Nippon Zeon Co., Ltd. for supplying the V-BR (70.4) and to Mr. Y. Fujita of Hitachi Kasei Polymer Co., Ltd. for supplying used tackifier resins and for help about a peel strength measurement.

\section{REFERENCES}

1. O. Olabishi, L. M. Robeson, and M. T. Shaw, "Polymer-Polymer Miscibility," Academic Press, New York, N. Y., 1979.

2. S. Akiyama, T. Inoue, and T. Nishi, "Polymer Blends," CMC Press, Tokyo, 1979 \& 1981.

3. D. R. Paul and S. Newman, (Eds.), "Polymer Blends," Vols. 1 and 2, Academic Press, New York, N. Y., 1978.

4. M. A. Krenceski, J. F. Johonson, and S. C. Temin, J. Macromol. Sci., Rev. Macromol. Chem., C26, 143 (1986).

5. C. W. Hock, J. Polym. Sci., C, 3, 139 (1963).

6. F. H. Wetzel, Adhesives Age, 7, 28 (1964).

7. K. Fukuzawa and T. Kosaka, Nippon Setchaku Kyokaishi, 3, 1 (1967).

8. Y. Takashima and T. Hata, Nippon Setchaku Kyokaishi, 20, 143 (1984).

9. T. Nishi, Nippon Setchaku Kyokaishi, 23, 449 (1987).

10. S. Akiyama, Nippon Setchaku Kyokaishi, 20, 485 (1984).

11. H. Ushiki, Y. Kano, S. Akiyama, and Y. Kitazaki, Eur. Polym. J., 22, 381 (1986).

12. S. Akiyama, H. Ushiki, Y. Kano, and Y. Kitazaki, Eur. Polym. J., 23, 327 (1987).

13. S. Akiyama, H. Ushiki, and H. Sugiyama, Rep. Prog. Polym. Phys. Jpn., 31, 455 (1988).

14. S. Kawahara and S. Akiyama, Nippon Setchaku Gakkaishi, 26, 142 (1990).

15. A. Yoshioka, K. Komuro, A. Ueda, H. Watanabe, S. Akita, T. Masuda, and A. Nakajima, Pure and Appl. Chem., 58, 1967 (1986).

16. T. Kyu and J. M. Saldanha, Macromolecules, 21, 1021 (1988).

17. T. Hashimoto, J. Kumaki, and H. Kawai, Maclomolecules, 16, 641 (1983).

18. J. H. Cahn and J. E. Hilliard, J. Chem. Phys., 28, 
258 (1958).

19. J. H. Cahn, J. Chem. Phys., 42, 93 (1965).

20. P. G. de Gennes, J. Chem. Phys., 72, 4756 (1980).

21. P. Pincus, J. Chem. Phys., 75, 1996 (1981).

22. K. Binder, J. Chem. Phys., 79, 6387 (1983).
23. O. Yasuda. T. Ougizawa, T. Inoue, and K. Miyasaka, J. Polym. Sci., Polym. Lett. Ed., 21, 813 (1983).

24. T. Inoue, T. Kobayashi, T. Hashimoto, T. Tagami, and K. Miyasaka, Polym. Commun., 25, 148 (1984). 